\title{
ANÁLISIS DE UNA OLA DE CALOR EXTREMA EN LA REGIÓN SUBTROPICAL DE AMÉRICA DEL SUR
}

\author{
FEDERICO AUGUSTO NORTE ${ }^{1}$, MARCELO ENRIQUE SELUCHI ${ }^{2}$, \\ JORGE LUIS GOMES ${ }^{2}$, SILVIA CARMEN SIMONELLI ${ }^{1}$
}

\author{
${ }^{2}$ Programa Regional de Meteorología -PRM- IANIGLA - CONICET \\ Av. Dr. Adrián Ruiz Leal s/n Parque San Martín (5500) Mendoza, Argentina \\ E-mails: fnorte@prmarg.org, scs@prmarg.org \\ ${ }^{2}$ Centro de Previsão do Tempo e Estudos Climáticos (CPTEC) \\ Rodovia Pte. Dutra Km 39 Cachoeira Paulista (12630-000) - SP, Brasil \\ E-mails: seluchi@cptec.inpe.br, gomes@cptec.inpe.br
}

Recibido Abril 2006 - Aceptado Noviembre 2006

\begin{abstract}
RESUMO
Este trabalho analisa as condições meteorológicas que levaram à ocorrência de uma intensa onda de calor na região subtropical da América do Sul, com temperaturas máximas superiores aos $40^{\circ} \mathrm{C}$ imediatamente à leste dos Andes, na última semana de Janeiro de 2003. Para isso, os termos da Equação Termodinâmica foram avaliados a partir de simulações numéricas realizadas com o modelo regional Eta/CPTEC.

O forte aumento da temperatura foi provocado pelo aquecimento por compressão adiabática, produto da subsidência, e pelo aquecimento superficial imediatamente a leste dos Andes. A análise da Equação Omega Quase-Geostrófica permitiu estabelecer que a subsidência de grande escala se deveu fundamentalmente ao avanço de uma crista de onda longa nos níveis meios da atmosfera. Além disso, as observações e as simulações numéricas apontam para a ocorrência de subsidência forçada (vento Zonda) a leste dos Andes, associada ao deslocamento de um cavado de onda curta imerso na crista de onda longa, à aproximação de uma frente fria e ao deslocamento para o sul da Baixa Termo-orográfica. Esse fato, muito pouco freqüente no verão, em combinação com os processos descritos anteriormente, explicaria a ocorrência de recordes históricos de temperatura no oeste da Argentina.
\end{abstract}

Palavras-chave: Onda Calor, Eta/CPTEC, Simulações, Eq. Termodinâmica.

\begin{abstract}
ANALYSIS OF AN EXTREME HEAT WAVE OVER THE SUBTROPICAL REGION OF SOUTH AMERICA

This work analyzes the meteorological conditions that caused the occurrence of an intense heat wave over subtropical South America, with temperatures higher than $40^{\circ} \mathrm{C}$ immediately to the east of the Andes, during the last week of January 2003. To that purpose the terms of the thermodynamic equation were evaluated through numerical simulations performed with the Eta/CPTEC regional model.

The strong temperature increase was caused by adiabatic warming (subsidence) as well as by the net positive surface heat flux, especially to the east of the Andes. The qualitative analysis of the Quasi-Geostrophic Omega Equation revealed that the large-scale subsidence was the result of the cold horizontal temperature advection and the advance of a wide ridge at midlevels. Additionally, the presence of forced orographic subsidence (Zonda wind) is evident from observations and numerical simulations in connection with the passage of a short trough immerse in the wider ridge, the approach of a cold front and the southward progress of the thermal-orographic low. Since this phenomenon is quite uncommon during summer, its incidence in combination to the other processes previously described would explain the occurrence of extreme maximum temperatures over western Argentina.
\end{abstract}

Keywords: Heat Wave, Eta/CPTEC, Simulations, Thermodynamic Eq. 


\section{INTRODUCCIÓN}

En la última semana del mes de enero de 2003, una ola de calor afectó la porción subtropical de América del Sur, incluyendo el centro-norte de Argentina, el sur de Brasil, Uruguay, parte de Bolivia y Paraguay. Las temperaturas más extremas se observaron en la parte oeste de Argentina entre los 30 y $35^{\circ} \mathrm{S}$, conocida como región de Cuyo, donde se registraron valores superiores a los $40^{\circ} \mathrm{C}$. La ciudad de Mendoza (localizada sobre los $32^{\circ} \mathrm{S}$ junto a la ladera de los Andes) alcanzó a las 20:15 UTC (17:15 hora local) del día 30 de enero la temperatura máxima de $44.4^{\circ} \mathrm{C}$, ver Figura 1, que corresponde al record absoluto en la historia meteorológica de esta ciudad. Sin embargo, la temperatura más elevada en aquel día se registró en la vecina ciudad de San Juan (unos $100 \mathrm{~km}$ al norte de Mendoza) con $45.4^{\circ} \mathrm{C}$. Esta situación meteorológica ocurrió durante la realización del SALLJEX (Experimento de campo para el estudio de la corriente en chorro en capas bajas, Vera et al. 2006), lo que posibilitó la obtención de sondeos atmosféricos adicionales realizados específicamente para esa campaña de medición.

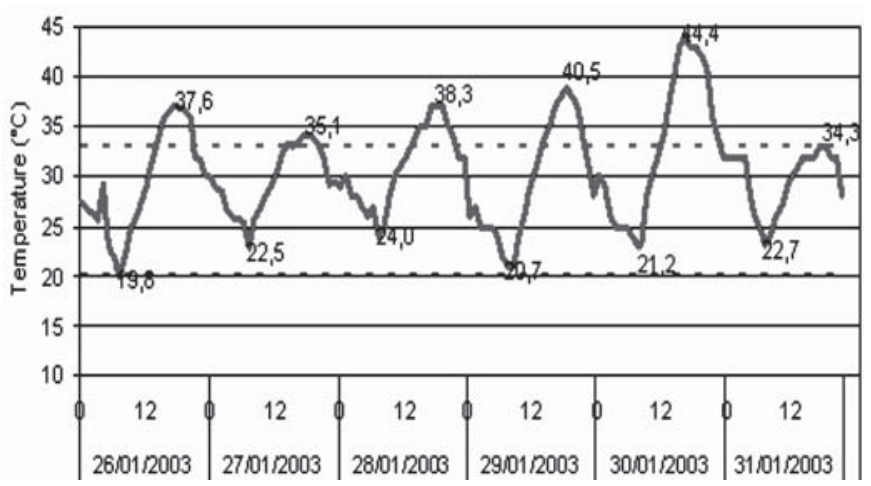

Figura 1 - Temperatura horaria $\left({ }^{\circ} \mathrm{C}\right)$ observada en la estación Mendoza $\left(32^{\circ} \mathrm{S}, 69^{\circ} \mathrm{O}\right)$ entre los días 26 y 31 de enero de 2003 . Los horarios corresponden a la hora local y los valores presentados en la parte superior (inferior) indican la temperatura máxima (mínima) registrada en cada día.

Las olas de calor suelen castigar con relativa frecuencia a la región subtropical de América del Sur durante todo el año, y en particular a la región de Cuyo. Norte et al (2000) encontraron que aquellas que ocurren en verano tienen una cierta recurrencia hacia fines del mes de diciembre (bautizada como "Ola de Calor de Navidad") y hacia la segunda quincena de enero, como en el caso analizado en este trabajo.

La ocurrencia de temperaturas máximas extremas constituye un peligro tanto para la salud de la población (golpes de calor), la agricultura (incendios, daños en cultivos), la hidrología (derretimiento de hielos, aluviones, avalanchas e inundaciones), el consumo y la distribución de energía, y la economía en general.
A pesar de los múltiples trastornos que suele causar este tipo de situación, resulta llamativa la escasez de trabajos abocados a este tema. Entre ellos se puede mencionar a Norte et al (2000) que, a partir de imágenes satelitales, analizaron las estructuras nubosas que suelen estar asociadas a la ocurrencia de olas de calor en el centro y el oeste de Argentina. Quizás el trabajo más completo en este sentido en la Argentina fue el realizado por Rusticucci y Vargas (1995), quienes estudiaron estadísticamente el comportamiento de la temperatura de superficie, y definieron un criterio para la detección de olas de frío y calor a partir de su desviación estándar. Por medio de un criterio estadístico de selección, estos autores analizaron algunas situaciones sinópticas particulares que definieron como "típicas". En este contexto, el extremo cálido de verano (enero de 1972) se caracterizó por el pasaje de un frente frío y el establecimiento de un centro de baja presión en el centro-oeste de Argentina, acompañado por un flujo del norte bien definido sobre la mayor parte de ese país. Esta situación fue denominada como "Tipo A". La situación de "Tipo B" está ligada a la actuación de un anticiclón de bloqueo sobre el Océano Atlántico, y una extensa área de baja presión sobre el centro-norte de Argentina. Por otra parte, Campetella y Rusticucci (1998) estudiaron la situación sinóptica que en marzo de 1980 condujo al record de temperatura máxima para ese mes, desde 1901, en la ciudad de Buenos Aires. La ola de calor se debió al predominio de una cuña de onda larga en la troposfera media y alta centrada en $55^{\circ} \mathrm{W}$, y a la persistencia del viento del sector noroeste en capas bajas sobre el norte y centro de la Argentina. En forma más indirecta, Alessandro (2003, 2005), Mendes et al. (2005) y Padilha (2006) analizaron estadísticamente la influencia que los bloqueos atmosféricos, tanto los que se ubican en el Océano Atlántico como en el Pacífico, ejercen sobre la precipitación y la temperatura en vastas regiones de Sudamérica. Esos autores señalan que dependiendo de la posición de los anticiclones de bloqueo, éstos pueden causar fuertes anomalías positivas de temperatura y negativas de lluvia.

El objetivo de este trabajo es analizar las condiciones meteorológicas dominantes durante la ola de calor que afectó la porción subtropical de América del Sur en la última semana de enero de 2003, desde un punto de vista sinóptico, dinámico y termodinámico, con el propósito de determinar los procesos físicos que contribuyeron al aumento de las temperaturas.

\section{DATOS Y METODOLOGÍA}

El diagnóstico de la situación sinóptica fue realizado a partir de los reanálisis del National Center for Environmetal Prediction (NCEP) (Kalnay et al. 1986), así como de datos de superficie y de altura derivados del Sistema Global de Telecomunicaciones. 
Los procesos físicos que condujeron a la ola de calor fueron analizados a través de simulaciones numéricas realizadas con el modelo Eta/CPTEC. Este modelo corre en forma operativa en el CPTEC desde mediados de 1996 dentro de un dominio que abarca prácticamente la totalidad del continente sudamericano, desde $12^{\circ} \mathrm{N}$ a $50^{\circ} \mathrm{S}$ y de $83^{\circ} \mathrm{O}$ a $26^{\circ} \mathrm{O}$. El Eta/CPTEC utiliza un retículo del tipo $\mathrm{E}$ de Arakawa y posee una física completa con parametrizaciones para la precipitación de escala sinóptica en forma explícita (Zhao et. al. 1991) y de escala convectiva según el esquema de Betts y Miller (1986) modificado por Janjic' (1994). Los intercambios turbulentos son representados mediante una actualización del esquema de Mellor y Yamada (1974) de orden 2.5, mientras que el esquema de radiación fue desarrollado por Lacis y Hansen (1974) y Fels y Schwarztkopf (1975). Los procesos de superficie son resueltos por el modelo de la Universidad del Estado de Oregon (OSU), (Chen et al. 1996), que es básicamente el acople del modelo de evapotranspiración potencial de Mahrn y Ek (1984), el de suelo de Mahrn y Pan (1984) y el de canopia de Pan y Mahrn (1987). Dicho modelo consta de 3 capas sub-superficiales y otra en donde se representa la canopia vegetal. La temperatura de superficie se determina a través de una ecuación de balace de energía lineal (Mahrn y Ek, 1984) que representa el efecto combinado de la superficie y la vegetación. Los flujos de calor son controlados por una ecuación de difusión, en donde la capacidad calórica y la conductivadad son funciones del contenido de agua en el suelo. Para más detalles sobre la física y las características generales de este modelo se puede consultar, por ejemplo, a Mesinger (1984), Black (1994) o Seluchi y Chou (2001), entre otros.

En el presente caso, el espaciamiento horizontal del retículo fue de $40 \mathrm{~km}$ y se utilizaron 38 niveles verticales irregularmente espaciados, con mayor densidad en la baja troposfera (separación mínima de $80 \mathrm{~m}$ ) y en las proximidades de la tropopausa (separación mínima de $200 \mathrm{~m}$ ). La simulación numérica fue iniciada a las 00 UTC del día 27 de enero y culminada a las 00 UTC del día 1 de febrero. De acuerdo a la Figura 1, este período cubre la evolución de la ola de calor, cuyo inicio puede establecerse el día 27 de enero, y que alcanza su mayor intensidad en la tarde del día 30 (en torno de las 20 UTC, 17 hora local).

La contribución relativa de cada proceso físico fue cuantificada a través del análisis de la ecuación termodinámica, aplicada a las simulaciones numéricas. La ecuación termodinámica en el modelo Eta/CPTEC (Mesinger et al 1988), se escribe como:

$$
\frac{\partial \mathrm{T}}{\partial \mathrm{t}}=-\overrightarrow{\mathrm{V}} \bullet \nabla_{\eta} \mathrm{T}-\dot{\eta} \frac{\partial \mathrm{T}}{\partial \eta}+\frac{\chi \mathrm{T} \omega}{\mathrm{p}}+\frac{\dot{\mathrm{Q}}}{\mathrm{c}_{\mathrm{P}}}
$$

donde T es la temperatura, $\overrightarrow{\mathrm{V}}$ el vector viento horizontal, $\omega$ la velocidad vertical en coordenadas isobáricas, $\chi=\mathrm{R}\left(\mathrm{c}_{\mathrm{p}}\right)^{-1}$; $\dot{\eta}$ es la velocidad vertical en coordenadas $\eta$ y $\dot{\mathrm{Q}} / \mathrm{C}_{\mathrm{P}}$ representa las fuentes y sumideros diabáticos de calor.

La ecuación (1) expresa que la tendencia local de la temperatura se debe al efecto de la advección horizontal (primer término de la derecha), al de la estabilidad estática (la advección vertical más la componente adiabática) representado por el segundo y tercer término de (1) y a los calentamientos/ enfriamientos diabáticos. Estos últimos fueron separados teniendo en cuenta la contribución individual de los procesos húmedos (liberación o consumo de calor latente debido a la convección y/o condensación/evaporación de gran escala), procesos radiativos atmosféricos y procesos de superficie (resueltos por el modelo OSU, previamente descrito). La difusión horizontal, despreciable frente a los otros efectos, fue incluida dentro del último término de (1).

Como en Seluchi et al (2003) cada uno de los términos del lado derecho de (1) fue cuantificado directamente a partir del código del modelo para cada paso de tiempo (96s) y grabado en archivos separados cada 3 horas. De esta forma se evita la aparición de residuos numéricos que enmascaran u obscurecen el aporte de los distintos términos, hecho que constituye uno de los principales problemas en la cuantificación de ecuaciones de balance a partir de salidas de modelos numéricos.

La ecuación Omega quasi-geostrófica también fue utilizada para diagnosticar las causas de los movimientos verticales.

\section{RESULTADOS}

\subsection{Diagnóstico y breve descripción sinóptica}

La Figura 2 muestra los datos "synop" correspondientes a la hora 21 UTC (18 hora local) del día 30 de enero de 2003. En general pueden observarse temperaturas muy elevadas, superiores a $\operatorname{los} 35^{\circ} \mathrm{C}$, en toda la parte subtropical de América del Sur. En particular, varias estaciones situadas en las proximidades de la ladera oriental de los Andes (como Mendoza y San Juan) presentan temperaturas superiores a los $40^{\circ} \mathrm{C}$. Estos valores contrastan fuertemente con los detectados en latitudes tropicales, por ejemplo en los Estados de Paraná y San Pablo, donde los registros son bien inferiores a $30^{\circ} \mathrm{C}$.

La Figura 3 (construida a partir de los re-análisis del NCEP interpolados al dominio y resolución del modelo Eta/CPTEC) resume la situación sinóptica del día 27 de enero a las 12 UTC. En ella puede identificarse un frente frío aproximadamente sobre los $36^{\circ} \mathrm{S}$, representado por la línea gruesa en el panel a), mientras que sobre latitudes subtropicales predomina la circulación asociada al Anticiclón Subtropical del Atlántico y se insinúa la formación de la Baja TermoOrográfica del Noroeste Argentino (BNOA). El campo de viento y de temperatura potencial adiabática equivalente (TPAE) en 
$850 \mathrm{hPa}$ (Fig 3b) permite comprobar que el frente mencionado es relativamente débil, destacándose la presencia de una lengua caliente inmediatamente al este de los Andes. La Figura 3c evidencia un mínimo de humedad relativa en la atmósfera media $(700 \mathrm{hPa})$ entre los 26 y $35^{\circ} \mathrm{S}$, coherente con el avance de una cuña de onda larga en $500 \mathrm{hPa}$ centrada aproximadamente sobre $\operatorname{los} 100^{\circ} \mathrm{O}$ (Fig 3d). En latitudes tropicales puede identificarse una circulación ciclónica relativamente barotrópica, asociada a la Zona de Convergencia del Atlántico Sur (SACZ) que actuó durante la última semana de enero de 2003.

Durante los dos días siguientes (28 y 29 de enero, Figuras no mostradas) sobresale el establecimiento de una cuña de onda larga en $700 \mathrm{hPa}$, cuyo eje sobre el continente se centra sobre los $58-60^{\circ} \mathrm{S}$, así como el desplazamiento zonal de la zona frontal al sur de los $40^{\circ} \mathrm{S}$ y el ligero aumento de los vientos del norte y de la TPAE en el nivel de $850 \mathrm{hPa}$.

Durante el día 30 se desarrolla rápidamente la $\mathrm{BNOA}$, (Figura 4a) ubicándose un poco más al sur respecto de su posición normal (Lichtenstein 1980, Ferreira et al 2005), simultáneamente con el aumento del viento del norte y de las temperaturas en la troposfera baja (ver Fig 4b). El fuerte secamiento generalizado en $700 \mathrm{hPa}$ (Fig 4c) muy probablemente responde a la subsidencia generada por el predominio de la circulación anticiclónica en niveles medios. Esta subsidencia presenta un máximo relativo a sotavento de los Andes (figura 4d) que aparentemente responde a un efecto orográfico. Evidencias de este efecto también surgen del campo de viento en $700 \mathrm{hPa}$ que muestra un aumento en los vientos de oeste sobre la ladera oriental de los Andes. Durante ese día se nota además el avance de un nuevo frente frío (ahora bien delimitado por el campo de isotermas de TPAE), vinculado a una vaguada de onda corta (Fig 4d) que se desplaza al sur de los $35^{\circ} \mathrm{S}$. Este frente frío provocó el descenso de la temperatura (aproximadamente $10^{\circ} \mathrm{C}$ ) durante el día 31 de enero, determinando el fin de la ola de calor. A pesar de eso las temperaturas continuaron siendo elevadas en los días posteriores, con máximas de $30^{\circ} \mathrm{C}$ a $35^{\circ} \mathrm{C}$, al norte de $25^{\circ} \mathrm{S}$.

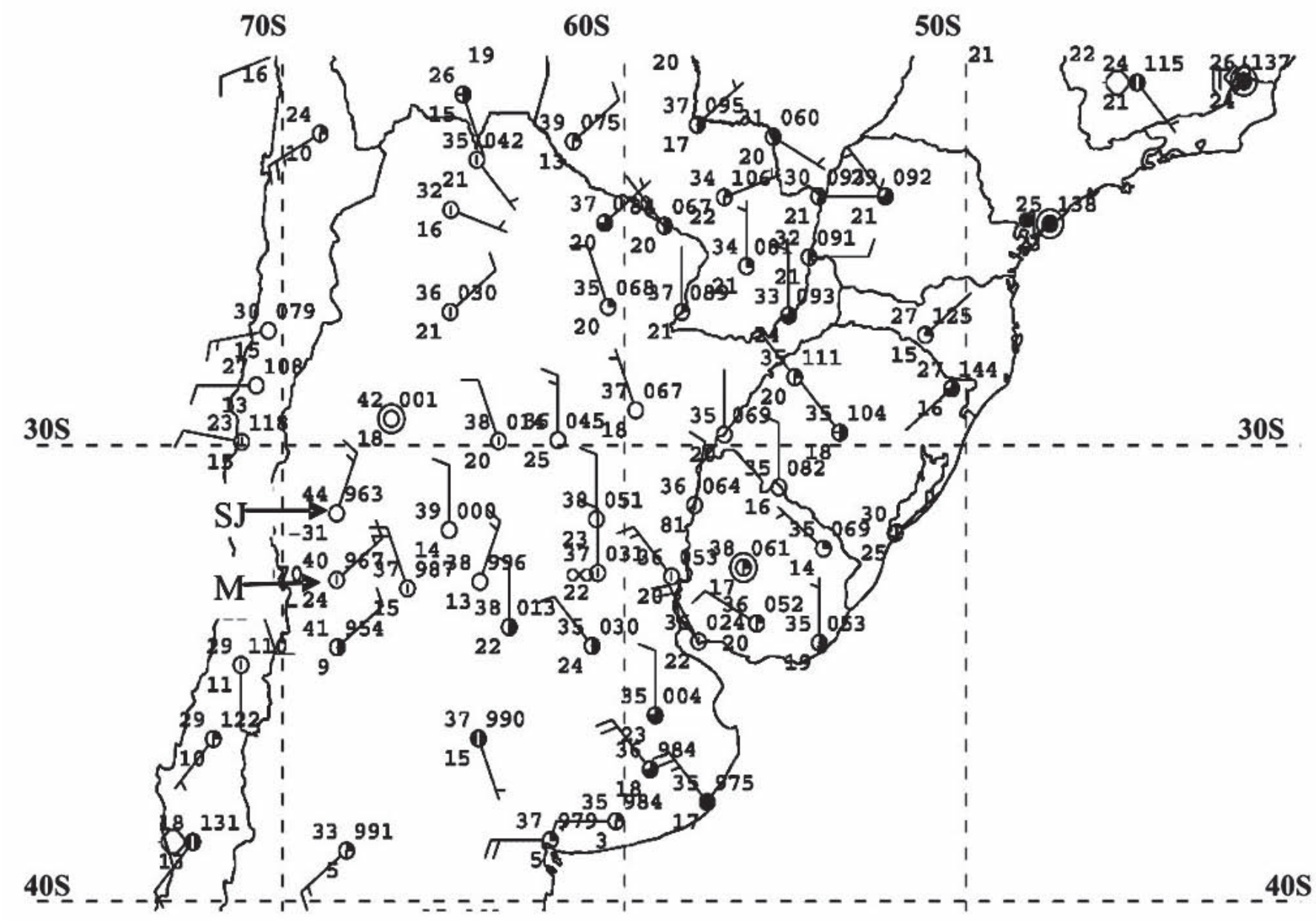

Figura 2 -: Datos "synop" correspondientes a la hora 21 UTC (18 hora local) del día 30 de enero de 2003. Las flechas con las iniciales M y SJ indican la ubicación de las estaciones Mendoza y San Juan, respectivamente. 
(a) SLP + T850 (27/01 12Z)

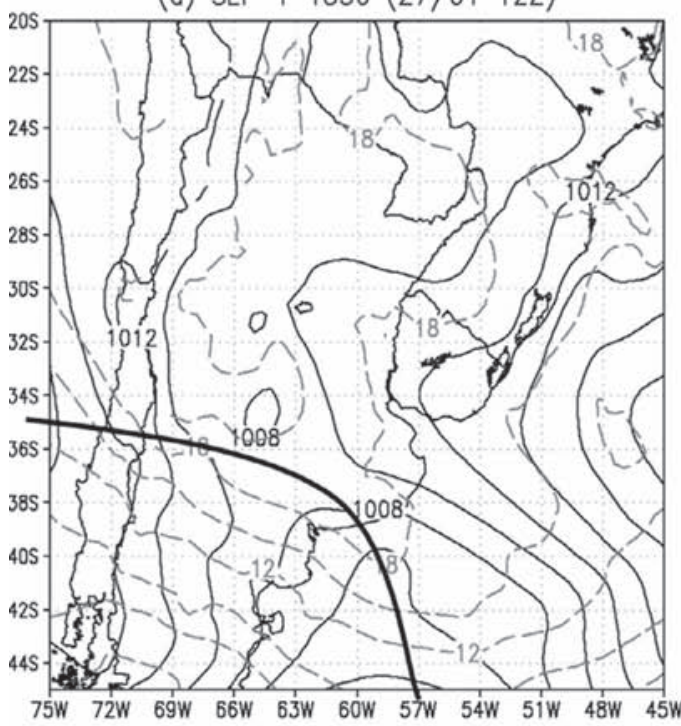

(c) HR+W 700

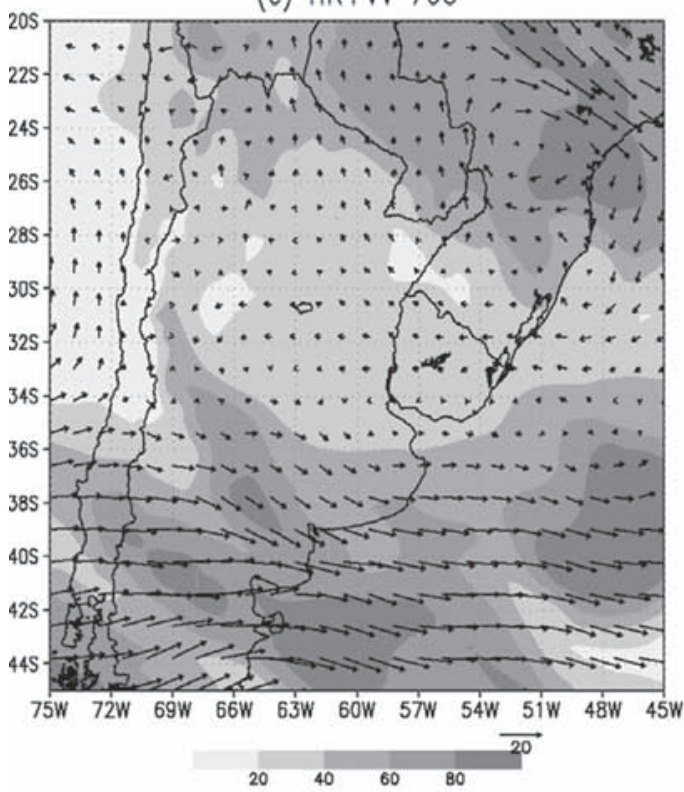

(b) Que+W 850

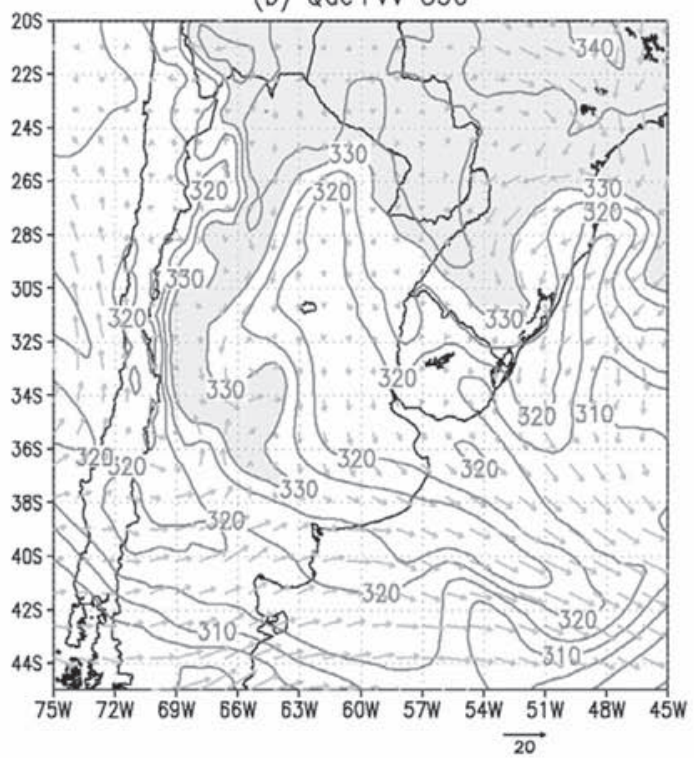

(d) $0 \mathrm{me}+\mathrm{Z}+\mathrm{T} 500$

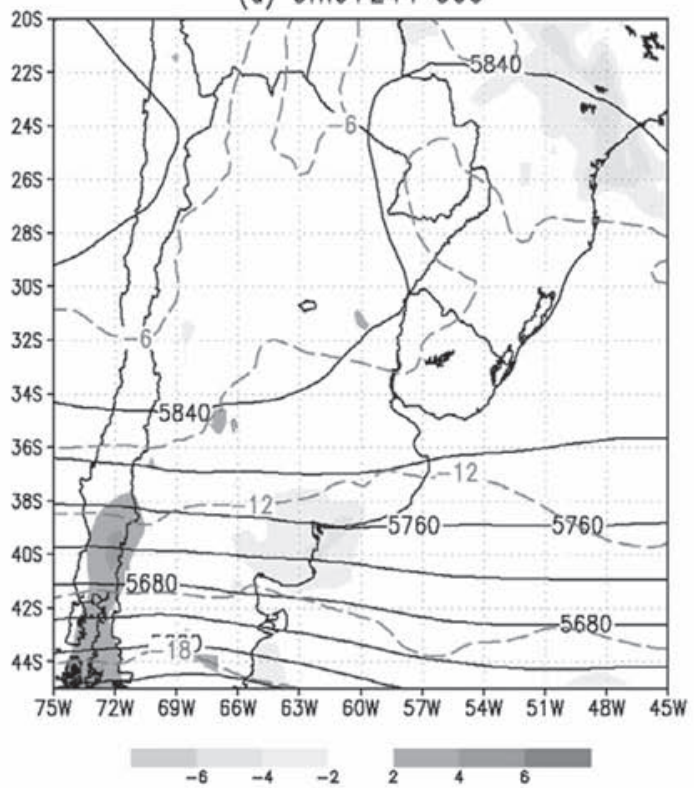

Figura 3 - Situación sinóptica del día 27 de enero de 2003 a las 12 UTC (re-análisis del NCEP interpolados a la resolución y dominio del modelo Eta/CPTEC). Panel a) presión reducida a nivel medio del mar (hPa, contornos) y temperatura en el nivel de $850 \mathrm{hPa}\left({ }^{\circ} \mathrm{C}\right.$, línea segmentada). La línea gruesa representa la posición aproximada del frente frío. Panel b): viento $\left(\mathrm{m} / \mathrm{s}\right.$, vectores) y temperatura potencial adiabática equivalente $\left({ }^{\circ} \mathrm{K}\right.$, contornos. Valores superiores a $330^{\circ} \mathrm{K}$ sombreados) en el nivel de $850 \mathrm{hPa}$. Panel c): Humedad relativa (\%, sombreado) y viento ( $\mathrm{m} / \mathrm{s}$, vectores) en el nivel de $700 \mathrm{hPa}$. Panel d) Omega ( $\mathrm{Pa} / \mathrm{s}$, sombreado), temperatura ( ${ }^{\circ} \mathrm{C}$, línea punteada) y geopotencial (mgp, contornos) en el nivel de $500 \mathrm{hPa}$. 
(a) SLP + T850 (31/01 00Z)

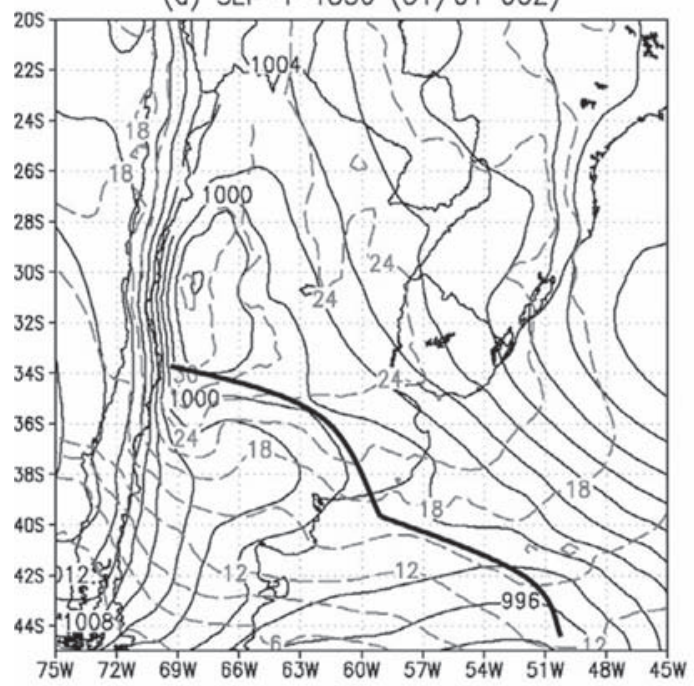

(c) $\mathrm{HR}+\mathrm{W} 700$

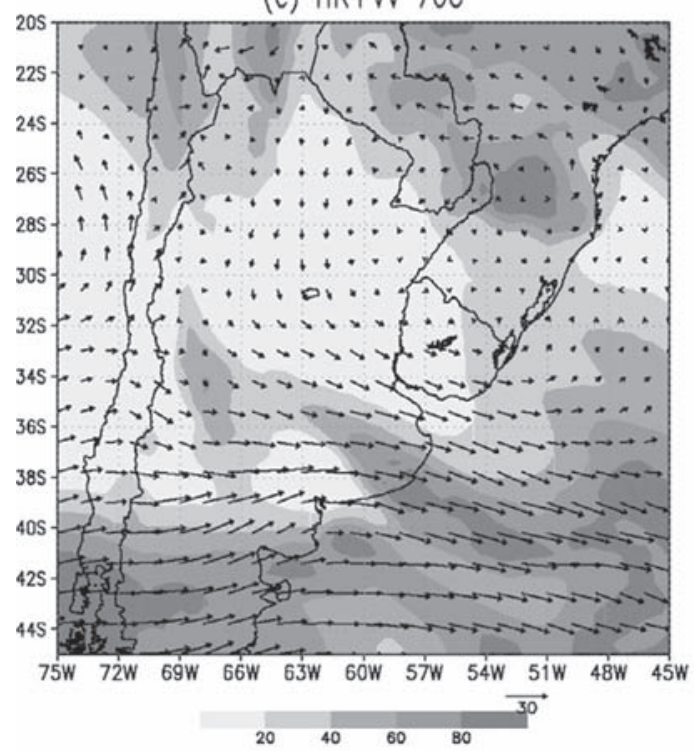

(b) Qae +W 850

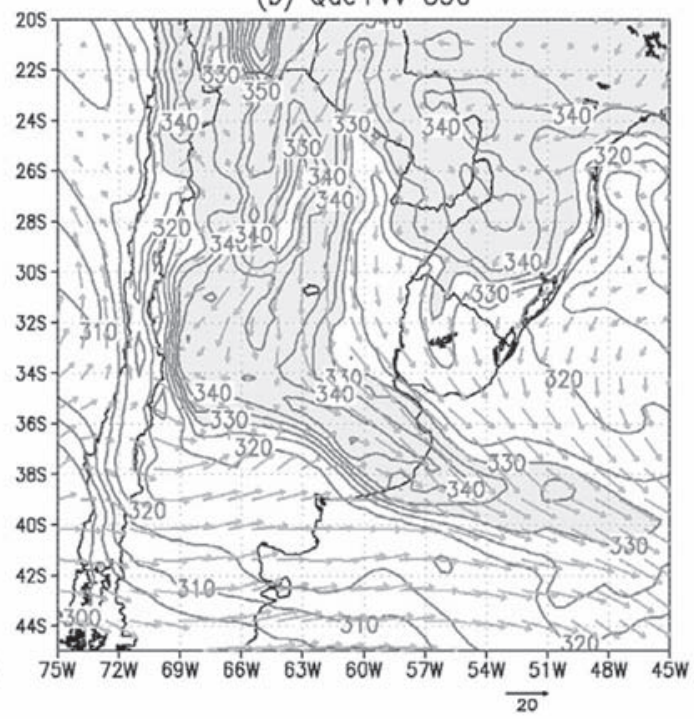

(d) $0 \mathrm{me}+Z+T 500$

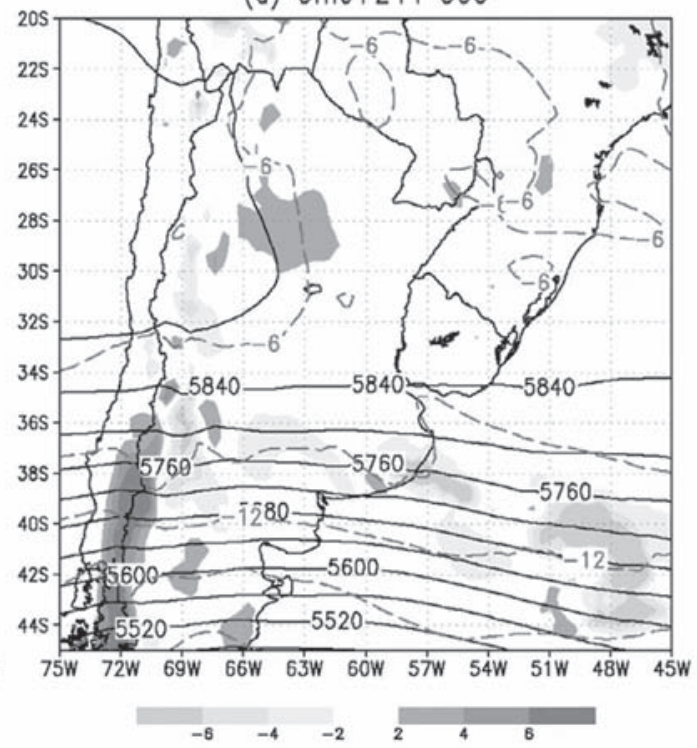

Figura 4 - Ídem Figura 3 para el día 30 de enero de 2003 a las 00 UTC.

\subsection{Validación de las simulaciones numéricas}

Un paso necesario hacia el entendimiento de los procesos físicos que provocaron la ola de calor, es la evaluación de las simulaciones realizadas con el Eta/CPTEC. La figura 5 presenta las temperaturas máximas simuladas por el modelo (líneas de trazos), que resultaron muy semejantes a las obtenidas a partir de la red sinóptica (superiores a $\operatorname{los} 45^{\circ} \mathrm{C}$ ) si bien el núcleo cerrado reproducido por el Eta/CPTEC parece situarse algo más al sur de su posición observada. Las tendencias de temperatura simuladas entre los días 27 y 30 de enero (sombreado), indican que las altas temperaturas están inmersas en un período de fuerte aumento térmico, restricto a la región subtropical de Sudamérica, y que no son el resultado de errores sistemáticos del modelo. El patrón y la magnitud de las tendencias simuladas resultan cercanos, con errores de aproximadamente $1^{\circ} \mathrm{C}$, a los medidos a partir de las estaciones sinópticas de superficie

Un examen mucho más exigente se presenta en la Figura 6, donde se comparan las marchas de las temperaturas observadas y simuladas en las dos ciudades que presentaron las máximas más extremas. En este análisis debe tenerse en cuenta que las ciudades de San Juan y Mendoza se encuentran a una 


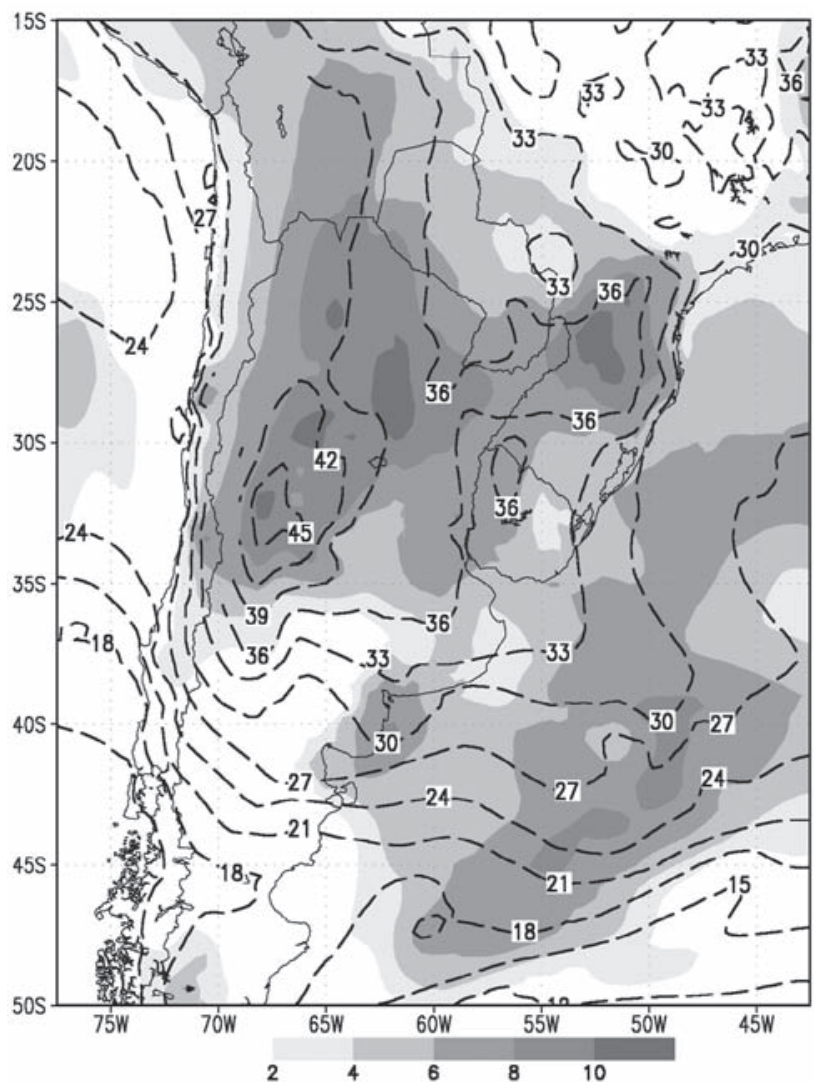

Figura 5 - Cambio de temperatura a 2 metros de altura simulado por el modelo Eta/CPTEC $\left({ }^{\circ} \mathrm{C}\right.$, sombreado) entre las 21 UTC de los días 27 y 30 de enero, y temperatura a 2 metros simulada a las 21 UTC del día 30 de enero de $2003\left({ }^{\circ} \mathrm{C}\right.$, línea punteada $)$.

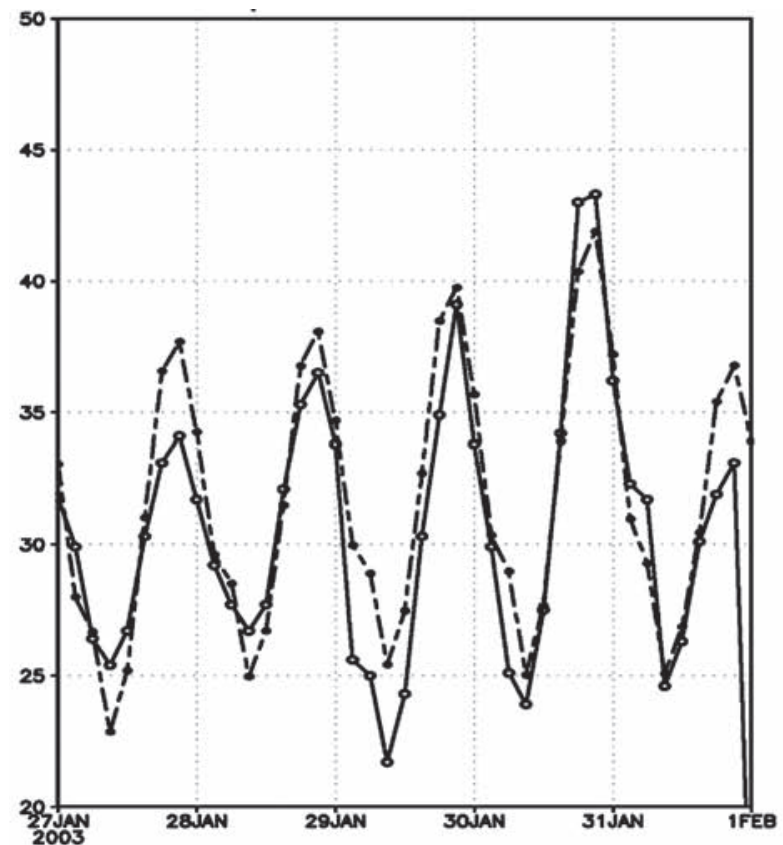

altura aproximada de 600-700m, muy cerca de la Cordillera los Andes (dos puntos de grilla del modelo) que presenta alturas cercanas a los $7000 \mathrm{~m}$. Este hecho dificulta reproducir fielmente lo que ocurre en una región geográfica tan fuertemente influenciada por la topografía. De todas formas la Figura 6 muestra que el modelo logra diagnosticar el aumento de la temperatura en ambas ciudades, así como el pico máximo, con aceptable precisión. Si bien existen algunas deficiencias en el inicio de la simulación, fundamentalmente la amplitud térmica en Mendoza y la temperatura máxima en San Juan, y también hacia el final de la integración numérica (subestimación del descenso térmico) la tendencia total de la temperatura entre los días 27 y 30 es bastante bien reproducida en ambas ciudades. Este es un factor importante, teniendo en cuenta la metodología utilizada en este trabajo.

\subsection{Diagnóstico de los procesos físicos}

La Figura 7 presenta un corte vertical, realizado en $32^{\circ} \mathrm{S}$ a partir de las simulaciones del Eta/CPTEC, de la tendencia de temperatura obtenida entre las 21 UTC del 27 de enero y las 21 UTC del 30 de enero. Según el Eta/CPTEC, el incremento térmico resulta mucho más acentuado y confinado a una capa más alta inmediatamente al este de los Andes. A partir de esta figura, se decidió integrar la ecuación termodinámica dentro de la capa comprendida entre superficie y $700 \mathrm{hPa}$, puesto que ahí se registraron las variaciones más significativas de temperatura.

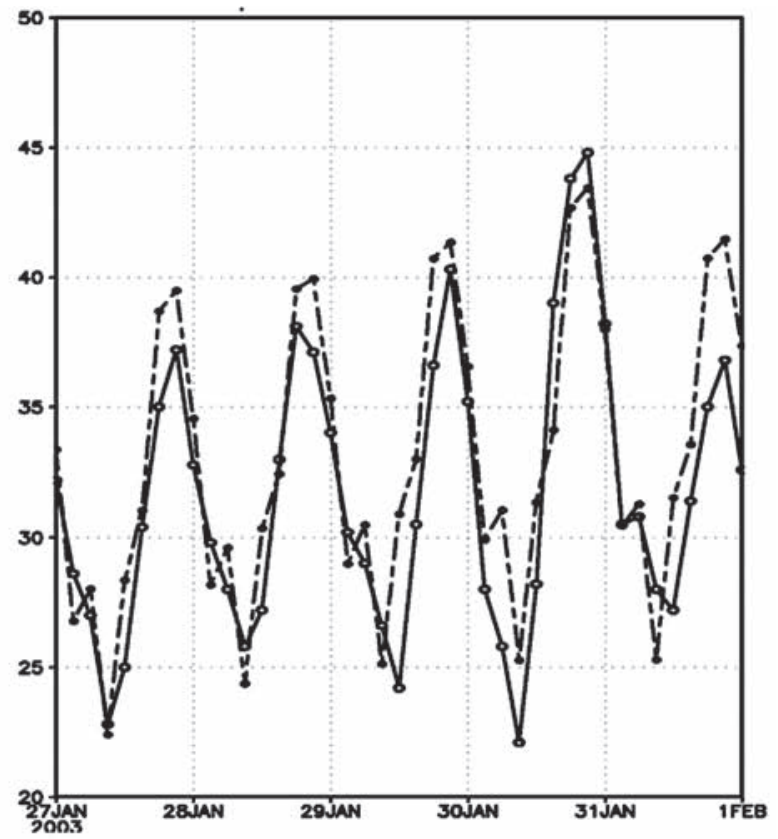

Figura 6 - Comparación entre la temperatura observada $\left({ }^{\circ} \mathrm{C}\right.$, línea continua) en las estaciones Mendoza (izquierda) y San Juan (derecha) con las simuladas por el modelo Eta/CPTEC $\left({ }^{\circ} \mathrm{C}\right.$, línea de guiones y puntos) 


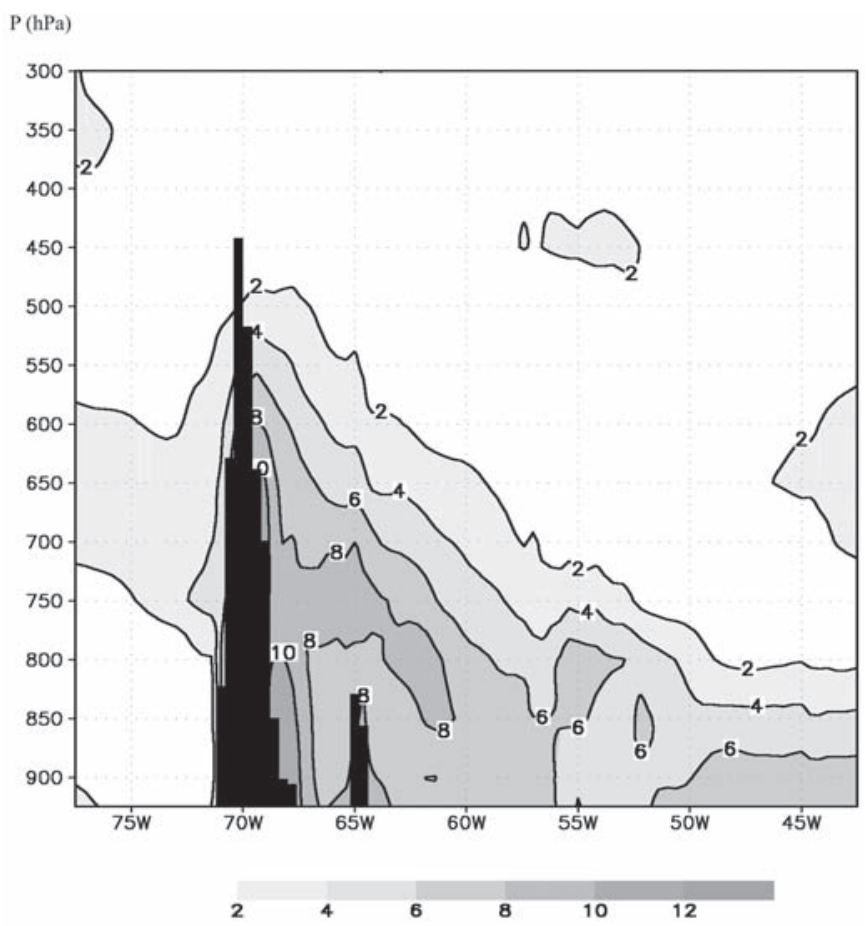

Figura 7 - Corte vertical realizado en $32^{\circ} \mathrm{S}$ de las tendencias de temperatura $\left({ }^{\circ} \mathrm{C}\right)$ simuladas por el modelo Eta/CPTEC entre las 21 UTC de los días 27 y 30 de enero de 2003. Las barras en color negro representa la topografia considerada por el modelo.

La Figura 8 exhibe el resultado de la integración de la ecuación termodinámica dentro de la capa $700 \mathrm{hPa} /$ superficie, entre las 21 UTC del día 27 de enero y la misma hora del 30 de enero. Contrariamente a lo que podría esperarse, la Fig 8a muestra que la advección térmica horizontal fue predominantemente fría o muy poco importante en toda el área subtropical de América del Sur, con máximos negativos sobre el sur de Brasil y sobre las planicies situadas inmediatamente al este de la Cordillera de los Andes. Las tendencias positivas de temperatura producto de la advección caliente se limitaron a las inmediaciones de la Provincia de Buenos Aires y al sector oceánico al sur de $35^{\circ} \mathrm{S}$. La Fig. $8 \mathrm{~b}$ permite evaluar el efecto de la estabilidad estática, que tuvo un papel destacado en la ocurrencia de la ola de calor. En general el patrón de las líneas de igual tendencia térmica es muy similar al de la Fig 8a pero con signo opuesto y módulo, en general, ligeramente mayor. Las tendencias positivas de temperatura provocadas por los movimientos verticales afectaron prácticamente la totalidad del área de estudio, con máximos relativos sobre la costa este de Rio Grande do Sul y en una estrecha franja localizada al Este de la Cordillera de los Andes, al norte de $32^{\circ} \mathrm{S}$. Al sur de esta latitud los enfriamientos y calentamientos debido a los movimientos verticales se alternan sucesivamente en franjas de aproximadamente $5^{\circ}$ de latitud, presentando signo contrario a un y otro lado de los Andes. Este último efecto se debe muy probablemente la interacción de la circulación zonal con la Cordillera de los Andes (Seluchi et al, 2006). El enfriamiento observado sobre la Provincia de Buenos Aires, probablemente ligado a la actividad frontal, es completamente enmascarado por el calentamiento producido por el efecto de la advección horizontal.

La Figura 8c presenta las tendencias de temperatura debidas a los procesos diabáticos, que incluyen los procesos radiativos en la atmósfera, el balance de calor en la superficie y los cambios de temperatura asociados a la liberación o consumo de calor latente. Dado que la pérdida de calor por radiación dentro de la capa analizada es relativamente uniforme en toda el área, y que el calentamiento superficial se limita a la región continental, sobre las áreas oceánicas la tendencia de temperatura es ligeramente negativa. Sobre la región continental comprendida entre los 40 y $25^{\circ} \mathrm{S}$, aproximadamente, el calentamiento superficial provoca un aumento de temperatura que es substancialmente más marcado inmediatamente al este de los Andes. Este factor que juega un rol importante en la ocurrencia de la ola de calor, se debe a la influencia de los flujos superficiales de calor. En efecto, la presencia de suelos arenosos y vegetación muy escasa (incluidos en el modelo de suelo utilizado) combinados con la ausencia de nubosidad, determinan flujos superficiales de calor sensible intensos y flujos de calor latente muy reducidos. La Figura 9, que muestra la imagen satelital promediada entre los días 26 y 30 de enero, permite comprobar que durante el período estudiado el cielo permaneció con muy poca o ninguna nubosidad, lo que contribuyó al fuerte calentamiento superficial. El panel "d" de la Figura 8, exhibe la contribución conjunta de los procesos considerados en la ecuación (1). Este campo de tendencias resulta idéntico al obtenido a partir de las salidas del modelo integradas dentro de la misma capa, ya que la metodología empleada presenta la ventaja de no generar residuos en el cálculo de los distintos términos. Un aspecto interesante es que si el mismo cálculo fuese efectuado en el nivel de $850 \mathrm{hPa}$, los resultados serían cualitativamente semejantes, pero con tendencias ligeramente más grandes. De la misma forma, también se obtienen valores proporcionalmente similares a los de la Figura 8 si se considera un período de tiempo más corto, como por ejemplo las 24 horas previas al máximo absoluto de temperatura. Por otro lado, si el mismo diagnóstico se realizase en un nivel más elevado, por ejemplo $700 \mathrm{hPa}$, aumenta la importancia de la estabilidad estática y disminuye la influencia de los procesos de superficie. Lo contrario ocurre si se elige un nivel más cercano al suelo para evaluar la ecuación (1). 
(a) Adv. Horiz

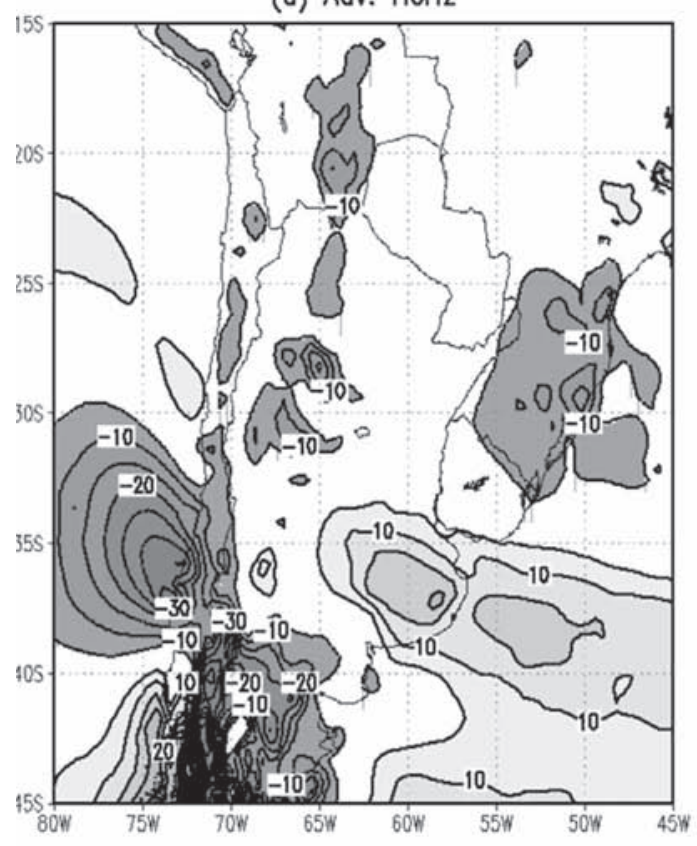

(c) Proc. Diab.

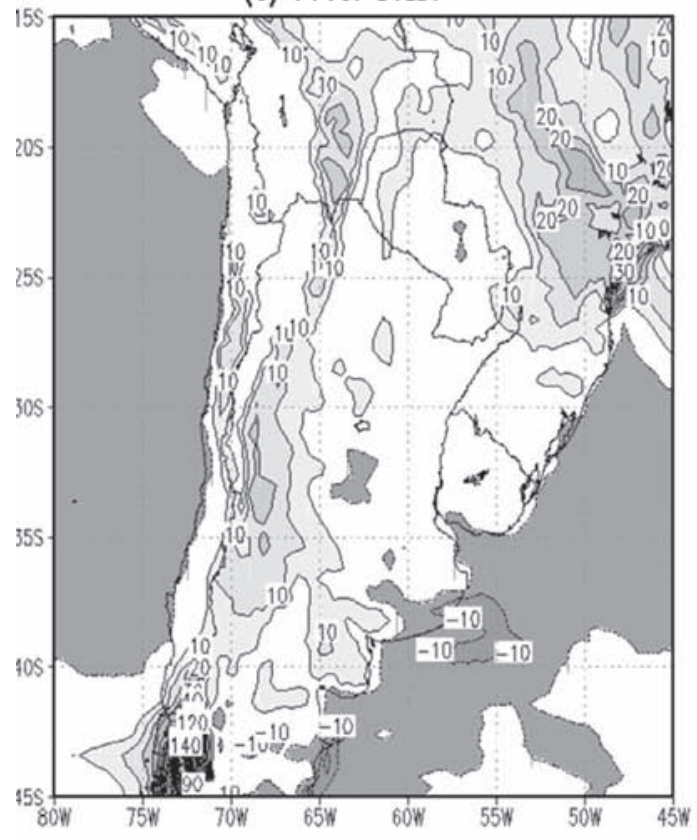

(b) Estab. Estat.

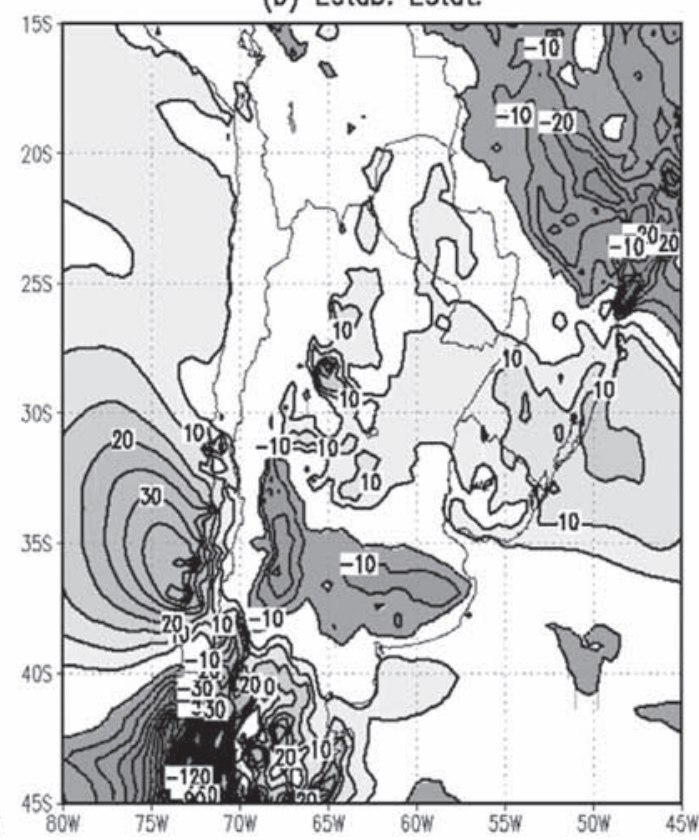

(d) Todos

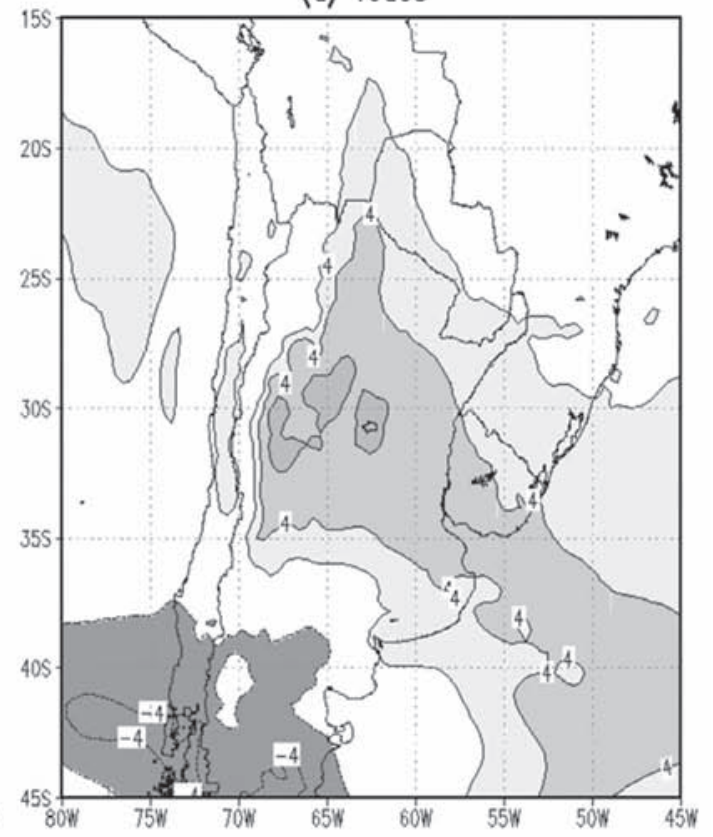

Figura 8 - Tendencias de temperatura $\left({ }^{\circ} \mathrm{C} / 3\right.$ días $)$ obtenidas a partir de los distintos términos de la ecuación termodinámica, entre las 21 UTC de los días 27 y 30 de enero, a partir de las simulaciones realizadas con el modelo Eta/CPTEC. Panel a): tendencias de temperatura $\left({ }^{\circ} \mathrm{C} / 3\right.$ días $)$ debido a la advección horizontal de temperatura. Panel b) tendencias de temperatura $\left({ }^{\circ} \mathrm{C} / 3\right.$ días) debido a la estabilidad estática (advección vertical más efecto adiabático). Panel c): tendencias de temperatura $\left({ }^{\circ} \mathrm{C} / 3\right.$ días) debido a los procesos diabáticos (procesos húmedos, radiativos y difusivos). Panel d): tendencias de temperatura $\left({ }^{\circ} \mathrm{C} / 3\right.$ días $)$ debido a la suma de los procesos considerados en los paneles a-c. 

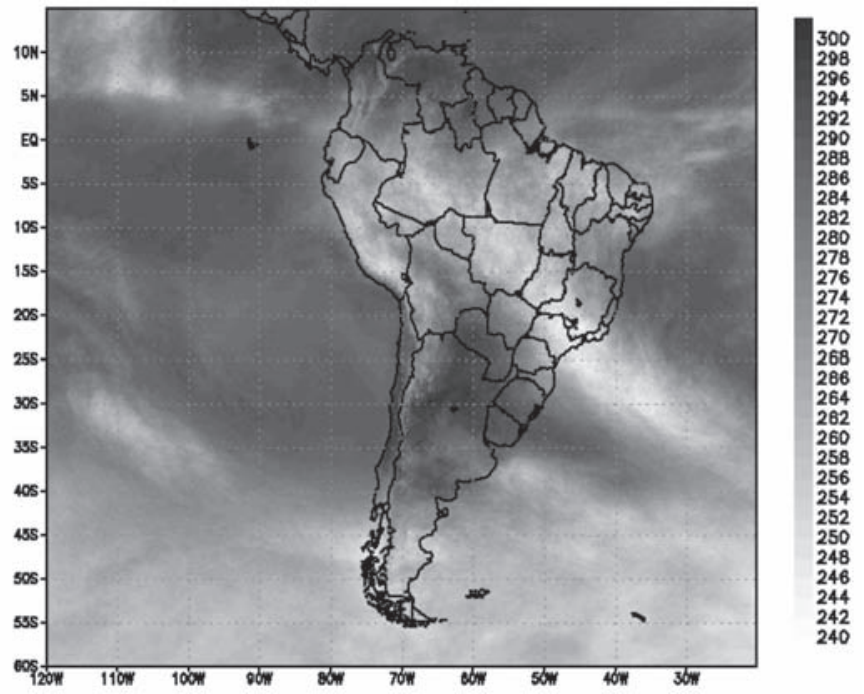

Figura 9 - Temperatura de brillo promedio $\left({ }^{\circ} \mathrm{K}\right.$, sombreado) entre los días 26 y 30 de enero de 2003.

\subsection{Diagnóstico del movimiento vertical}

El diagnóstico termodinámico anterior revela la importancia del movimiento vertical (subsidencia) como proceso causante de la ola de calor, pues aún el balance de calor en la superficie fue indirectamente influenciado por este efecto. La Figura 10, que presenta el campo de movimiento vertical en el nivel de $700 \mathrm{hPa}$ promediado entre el 27 y el 30 de enero, evidencia el predominio de la subsidencia al norte de los $32^{\circ} \mathrm{S}$, con un máximo localizado al este de la Cordillera de los Andes. En esta sección se realiza un diagnóstico de los procesos dinámicos que determinaron el predominio de los movimientos descendentes durante el período analizado. Para tal fin puede utilizarse la Ecuación Omega Quasi-gesotrófica (Bluestein 1993, Satyamurty 2004):

$$
\begin{aligned}
& \left.\left[\nabla^{2}+\left(\mathrm{f}_{\mathrm{o}}^{2} / \sigma\right)\left(\partial^{2} / \partial \mathrm{p}^{2}\right)\right\}\right] \Phi= \\
& \left(\mathrm{f}_{\mathrm{o}} / \sigma\right)(\partial / \partial \mathrm{p}) \mathrm{V}_{\mathrm{g}} \cdot \nabla\left(\left\{1 / \mathrm{f}_{\mathrm{o}}\right\} \nabla^{2} \Phi+\mathrm{f}\right)+ \\
& (1 / \sigma) \nabla^{2}\left[\mathrm{~V}_{\mathrm{g}} \cdot \nabla(-\partial \Phi / \partial \mathrm{p})\right]
\end{aligned}
$$

La ecuación (2) expresa que el signo del movimiento vertical dependerá de la variación con la altura de la advección de vorticidad geostrófica absoluta y del signo de la advección térmica (advección de espesor) por el viento geostrófico.

La Figura 11 ayuda a diagnosticar el signo de los dos términos de la derecha de (2): el panel de la izquierda (Fig. 11a) que muestra el campo de geopotencial en los niveles de $900 \mathrm{y}$ $500 \mathrm{hPa}$ promediados entre los días 27 y 30 de enero, permite inferir un aumento de la advección de vorticidad anticiclónica

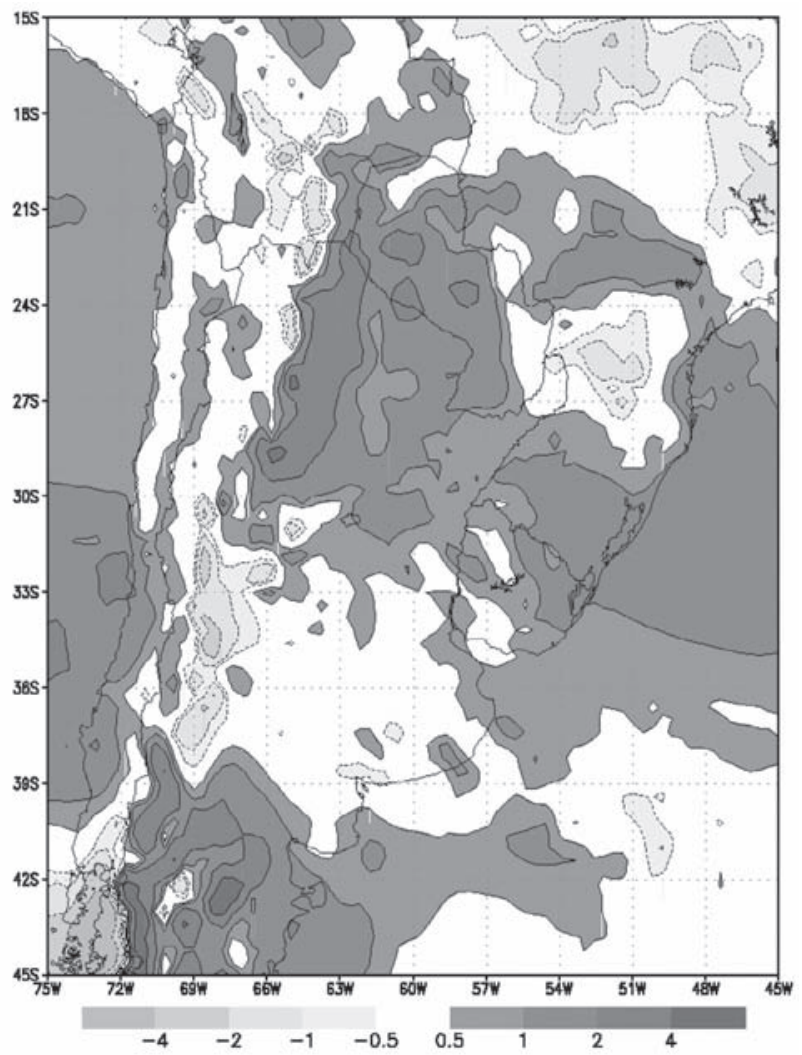

Figura 10 - Velocidad vertical Omega $(\mathrm{Pa} / \mathrm{s}$, sombreado) en el nivel de $700 \mathrm{hPa}$ promediada entre los días 27 y 30 de enero, a partir de las simulaciones del modelo Eta/CPTEC

con la altura, ya que el campo de superficie no sufrió variaciones significativas durante ese período mientras que una cuña de onda larga avanzó en los niveles más elevados. El panel de la derecha (Fig 11b) muestra que la advección horizontal fría sobre el área de estudio se debió a que el viento del este originado por el Anticiclón subtropical del Atlántico se superpuso a un campo térmico caracterizado por un gradiente prácticamente zonal. De esta manera, la única región que presentó advección caliente fue la afectada por vientos del sector noroeste, localizada en las inmediaciones de la Provincia de Buenos Aires. En síntesis, ambos términos de la ecuación (2), es decir el aumento de la vorticidad absoluta anticiclónica con la altura y la advección térmica fría, contribuyeron al aumento de la subsidencia. Sin embargo, un análisis cuantitativo de estos dos efectos (figuras no incluidas) permitió establecer que el primer término de la derecha (variación de la vorticidad geostrófica absoluta con la altura) explica prácticamente en forma total el campo de movimiento vertical observado. El efecto de la advección térmica tuvo un peso significativo (comparativamente igual) sólo en una estrecha franja centrada aproximadamente sobre los $38^{\circ} \mathrm{S}$, al este de los Andes. 


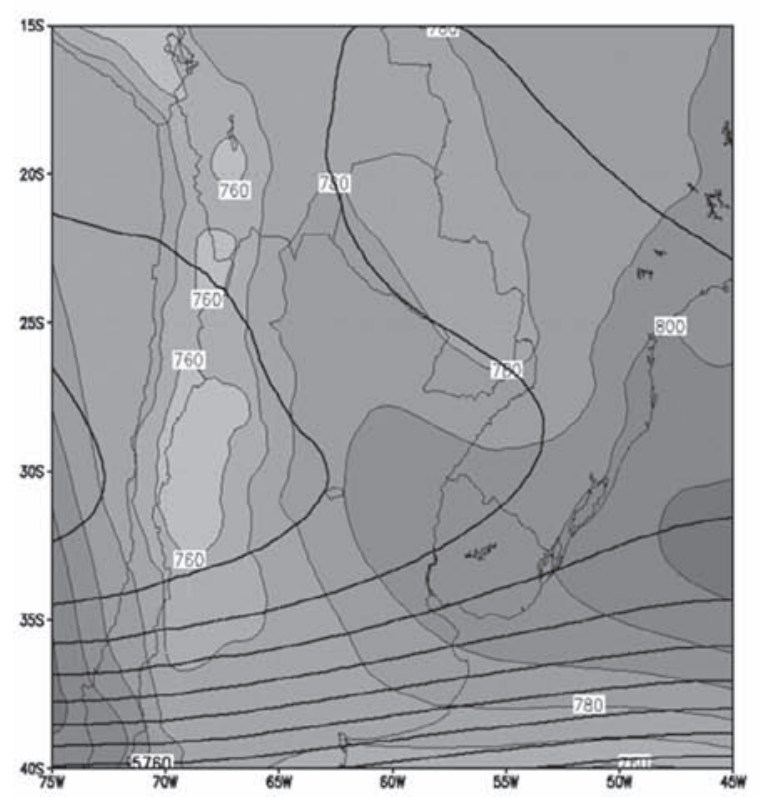

$\begin{array}{llllllllll}720 & 730 & 740 & 750 & 760 & 770 & 780 & 790 & 800 & 810\end{array}$

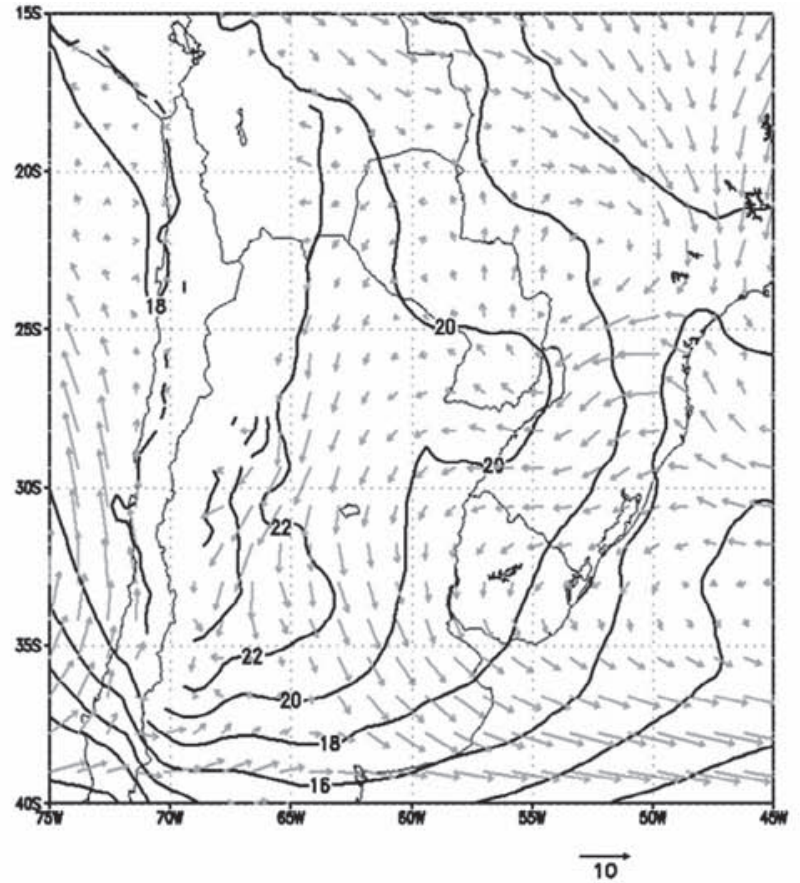

Figura 11 - (a) Altura geopotencial en el nivel de $900 \mathrm{hPa}$ (mgp, sombreado) y $500 \mathrm{hPa}$ (mgp, contornos) promediados entre los días 27 y 30 de enero a partir de las simulaciones del modelo Eta/CPTEC. (b) Campo de viento (flechas) e isotermas (contornos) en el nivel de $850 \mathrm{hPa}$ promediados entre los días 27 y 30 de enero a partir de las simulaciones del modelo Eta/CPTEC.

Además de los mecanismos mencionados en (2), generalmente responsables de los ascensos o descensos de escala sinóptica, existieron fuertes evidencias de la presencia de subsidencia orográfica sobre la Región de Cuyo, especialmente durante el día 30 de enero. Este fenómeno, conocido localmente como viento Zonda (Norte 1988, Seluchi et al 2003b) fue favorecido por el pasaje de una vaguada de onda corta inmersa en la cuña de onda larga, así como por la aproximación de un frente frío y el desplazamiento hacia el sur de la BNOA, que garantizó un fuerte gradiente bárico a través de los Andes (ver figura 4). Según Norte (1988) este último es uno de los principales elementos predictivos para determinar la ocurrencia de viento Zonda. La Figura 12 ilustra el sondeo de Mendoza, realizado a las 18 UTC de ese día (en el marco del Proyecto SALLJEX), en donde se puede apreciar un fuerte secamiento en los niveles bajos y medios de la tropósfera. En particular se detecta un gradiente vertical adiabático debajo de los $700 \mathrm{hPa}$, típico de la irrupción de viento Zonda. Por otra parte una inspección detallada de la Figura 1 revela que las altas temperaturas registradas sobre la región de Cuyo estuvieron acompañadas por temperaturas de rocío extremadamente bajas $\left(-31^{\circ} \mathrm{C}\right.$ en San Juan, $-24^{\circ} \mathrm{C}$ en Mendoza y $9^{\circ} \mathrm{C}$ en Malargüe) en comparación con otras estaciones situadas a la misma latitud.
FNL Archive

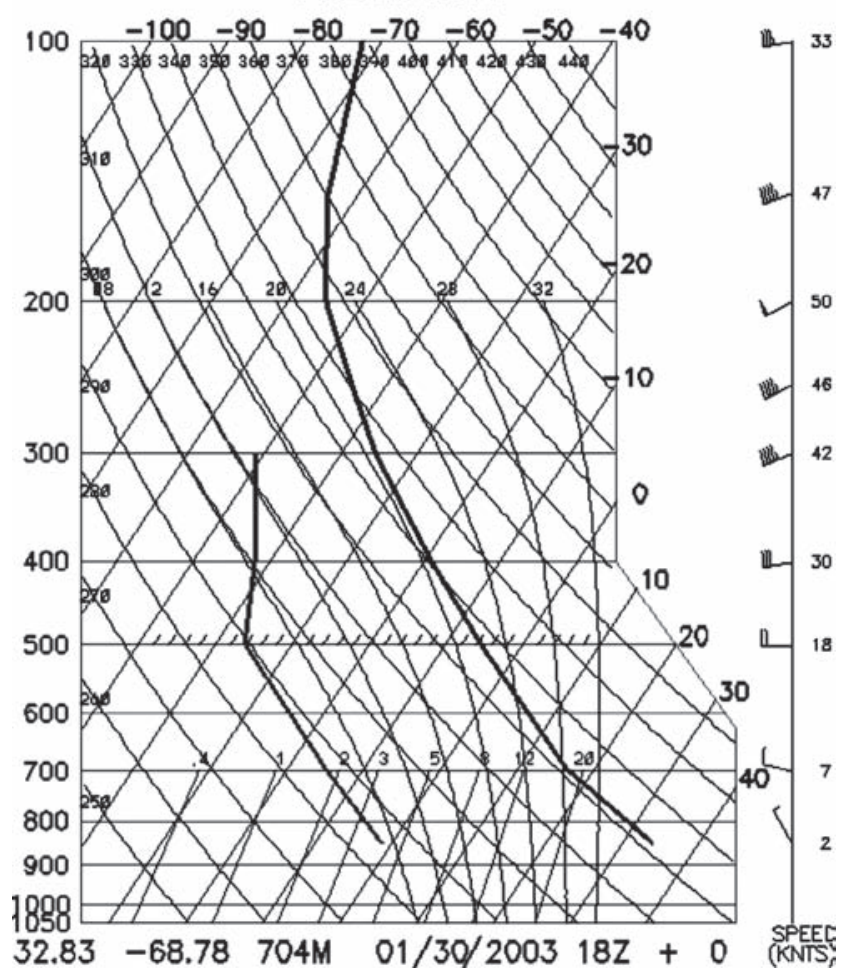

Figura 12 - Skew-T correspondiente a la estación Mendoza a las 18 UTC (15 hora local) del día 30 de enero de 2003. 
Las simulaciones numéricas realizadas con el modelo Eta también sugieren la ocurrencia de viento Zonda, tal como se observa en la Figura 13, que muestra un máximo relativo de subsidencia inmediatamente al este de los Andes, que contrasta fuertemente con el ascenso detectado a barlovento. Estudios previos (Seluchi et al., 2003b) mostraron que el modelo Eta/CPTEC, aun utilizando una resolución relativamente baja, es capaz de diagnosticar y pronosticar la ocurrencia de viento Zonda con suficiente exactitud. El número de Froude obtenido sobre los $32^{\circ} \mathrm{S}$ a partir de las simulaciones numéricas, definido como: $\mathrm{Fr}=\frac{\mathrm{U}}{\mathrm{NH}}$ (donde U es la velocidad del viento perpendicular a obstáculo orográfico, H la altura de la montaña "vista" por cada nivel de presión y $\mathrm{N}$ la frecuencia de Brunt-Väisälä), presenta un fuerte aumento durante el día 30 (figura no incluida). Efectivamente, el acercamiento de la vaguada de onda corta provocó la disminución de la estabilidad estática y el aumento del viento en los niveles medios y altos de la tropósfera. Los números de Froude superaron la unidad en el nivel de $700 \mathrm{hPa}$ y se aproximaron a uno en $850 \mathrm{hPa}$, lo que demuestra que las condiciones atmosféricas en ese momento eran favorables para que el aire situado sobre el Océano Pacífico pudiera atravesar la Cordillera de los Andes.

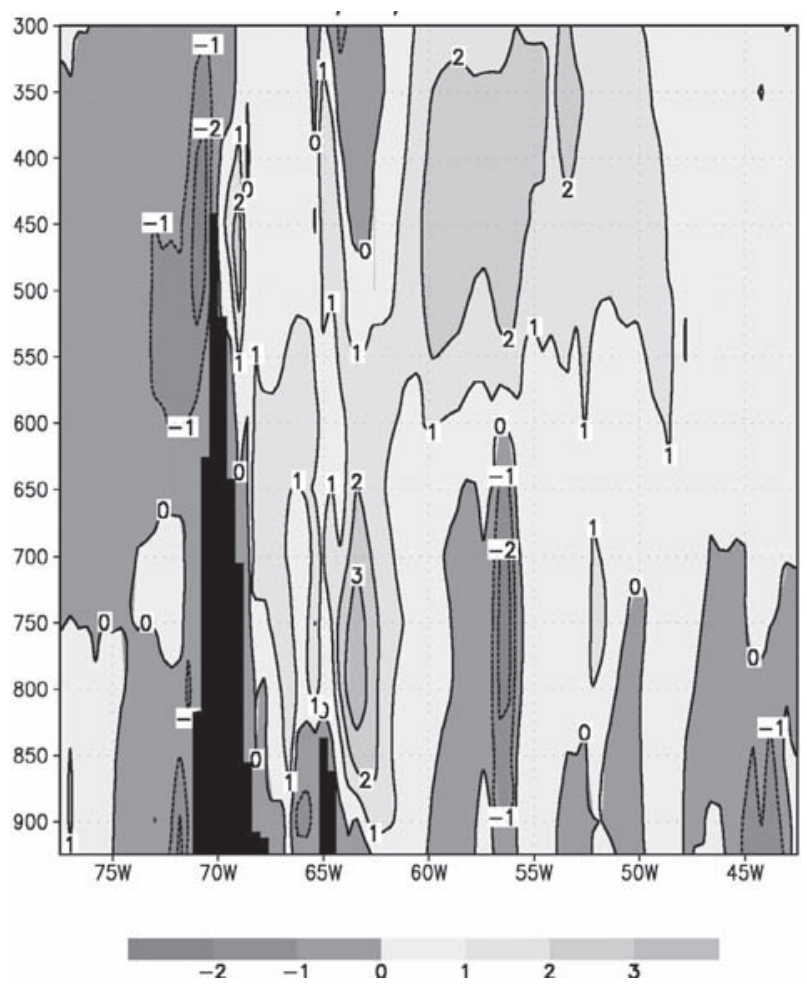

Figura 13 - Corte vertical de Omega $(\mathrm{Pa} / \mathrm{s})$ realizado en $32^{\circ} \mathrm{S}$ a las 21 UTC del día 30 de enero a partir de las simulaciones del modelo Eta/ CPTEC. Las barras en color negro representa la topografia considerada por el modelo.

\section{CONCLUSIONES}

Durante la última semana de enero de 2003 se registró una intensa ola de calor que provocó temperaturas extraordinariamente elevadas en la región subtropical de América del Sur, con máximos absolutos sobre la región de Cuyo (oeste de Argentina). En la ciudad de Mendoza se alcanzó la temperatura máxima histórica de $44.4^{\circ} \mathrm{C}$, alrededor de las 17 hora local del 30 de enero, mientras que simultáneamente en la ciudad de San Juan se registró la temperatura más elevada de este episodio, con $45.4^{\circ} \mathrm{C}$. Las temperaturas comenzaron a elevarse a partir del día 23 , pero lo hicieron con mayor intensidad a partir del 27 de enero y hasta el 30 de enero. Este último período fue analizado en el presente trabajo, con el objetivo de determinar los procesos físicos que determinaron la ola de calor.

La metodología empleada consistió en evaluar los términos de la ecuación termodinámica directamente del código del modelo regional Eta/CPTEC, con el objeto de evitar posibles residuos numéricos. Para tal efecto se realizaron simulaciones numéricas del período comprendido entre el 27 y el 31 de enero, y se analizaron las tendencias de temperatura diagnosticadas entre las 21 UTC del día 27 y la misma hora del día 30.

Contrariamente a lo que podría esperarse, y a lo encontrado en los antecedentes bibliográficos, la advección horizontal sobre la región de estudio fue predominantemente fría, debido la influencia de una circulación anticiclónica en la baja tropósfera centrada sobre los $58-60^{\circ} \mathrm{S}$, en combinación con isotermas dispuestas en sentido meridional. La advección caliente se restringió a las inmediaciones de la Provincia de Buenos Aires. El patrón de advecciones encontrado es relativamente semejante al descripto por Seluchi et al. (2003) quienes describieron los procesos de formación de la BNOA, también presente en la situación analizada.

El calentamiento por subsidencia y el balance de calor en superficie fueron los dos procesos que contribuyeron a la ola de calor. El primero actuó sobre casi toda el área de estudio, con más intensidad sobre el sur de Brasil y sobre la ladera este de los Andes, mientras que el segundo fue más significativo sobre la región de Cuyo debido a la combinación de cielo despejado, baja humedad y suelos casi desprovistos de vegetación. Sobre la Provincia de Buenos Aires hubo enfriamiento provocado por movimientos de ascenso, pero éstos no compensaron totalmente el calentamiento provocado por la advección horizontal.

El análisis de la Ecuación Omega Quasi-geostrófica permitió establecer que la subsidencia prevaleció en la troposfera baja y media sobre latitudes subtropicales, debido fundamentalmente al aumento con la altura de la advección de vorticidad anticiclónica. Este último mecanismo estuvo ligado al avance de una cuña de onda larga en los niveles medios de la atmósfera. En particular sobre la Región de Cuyo el avance 
de una vaguada de onda corta, la aproximación de un frente frío y el desplazamiento hacia el sur de la BNOA, favorecieron la ocurrencia de viento Zonda durante el día 30 de enero. Esto puede comprobarse a partir de las bajas temperaturas de rocío observadas inmediatamente al este de los Andes, de las simulaciones numéricas y, fundamentalmente, del análisis del sondeo de Mendoza. Según Norte (1988) la ocurrencia de viento Zonda en superficie no es frecuente en la estación estival. En este caso, su aparición durante un periodo relativamente corto, y su combinación con los procesos físicos mencionados en el párrafo anterior, motivaron la ocurrencia de temperaturas máximas históricas inmediatamente al este de los Andes.

En general, el estudio de olas de calor presenta dificultades ya que estas situaciones suelen prolongarse durante varios días, y en muchas ocasiones los aumentos de temperatura son constantes pero pequeños. Este hecho, por un lado, perjudica la eficiencia de las simulaciones numéricas que deben extenderse en el tiempo y, por otro, dificulta el análisis de los términos de la ecuación termodinámica que pueden incluir efectos de magnitud pequeña y signo contrario. En este trabajo la metodología empleada resultó satisfactoria debido a que se analizó un caso intenso y relativamente corto (la temperatura aumentó prácticamente $10^{\circ} \mathrm{C}$ en 72 horas) en el cual los términos de la ecuación termodinámica fueron cuantificados directamente a partir del código del modelo.

Los resultados resaltan la importancia del calentamiento por subsidencia como mecanismo potencialmente importante para la ocurrencia de olas de calor. Este proceso es frecuentemente desestimado, pues en muchas ocasiones es difícil de ser cuantificado, especialmente cuando se intenta hacerlo a partir de campos analizados (reanálisis).

\section{AGRADECIMIENTOS}

Este trabajo fue parcialmente financiado por los proyectos PROSUL, proceso 490353/2004-5 del Conselho Nacional de Desenvolvimento Científico e Tecnológico (CNPq), y por la Fundação de Amparo à Pesquisa do Estado de São Paulo, FAPESP, a través del Proyecto SALLJEX-Brasil

\section{REFERENCIAS BIBLIOGRÁFICAS}

ALESSANDRO A.P. The Influence of Blocking Events on Temperature and precipitation in Argentina during the 1990s. Meteorologica, 2003 28, 39-52

ALESSANDRO A.P: Bloqueos Simultáneos em el Atlántico y Pacífico Sur y sus Influencias sobre la República Argentina, Revista Brasileira de Meteorología, 2005, 277-300.
BETTS A.K., MILLER M.J., A new convective adjustment scheme. Part II: Single column test using GATE wave, BOMEX, and arctic air-masses data sets Quart. J. Roy. Meteor. Soc., 112, 1986, 1306-1335.

BLACK T. L., The new NMC mesoscale Eta model: Description and forecast examples. Wea. Forecasting, 9, 1994, 256-278.

BLUESTEIN H. B., Synoptic-dynamic meteorology in midlatitudes, Volume II: Observations and theory of weather systems. Oxford University Press, 1993, 953 pp.

CAMPETELLA, C., M. RUSTICUCCI, Synoptic analysis of an extreme heat wave over Argentina in March 1980. Meteorological Applications 5, 1998, 217-226.

CHEN F. et al, Modeling of land surface evaporation by four schemes and comparison with FIFE observations, J. Geoph. Res., III, NºD3, 1996, 7251-7268.

FELS, S. B., SHWARZKOPF M. D., The simplified exchange approximation: A new method for radiative transfer calculations. J. Atmos. Sci., 32, 1975, 1475-1488.

FERREIRAL., et al., Estructura de los patrones termodinámicos asociados a eventos de Baja del Noroeste Argentino, Anales del CONGREMET IX, Buenos Aires, Argentina, 2005.

JANIC', Z.I., The new NMC mesoscale Eta model: Description and forecast examples. Wea. Forecasting,9, 1994, 265-278.

Kalnay, E. et al, The NCEP/NCAR 40-year Reanalysis Project. Bulletin of the American Meteorological Society 77 1996, 437-472

LACIS, A. A., J. E. HANSEN, A parameterization of the absorption of solar radiation in the earth's atmosphere. J. Atmos. Sci., 31, 1974, 118-133.

LiChtenstein, E. R., La Depresión del Noroeste Argentino, 1980, 130p.. Dissertação de Doutorado em Meteorologia. Departamento de Meteorologia, Facultad de Ciencias Exactas y Naturales Universidad de Buenos Aires, Argentina.

MAHRT L., K. EK, The influence of atmospheric stability on potential evaporation. J. Clim. Appl. Meteorol., 23, 1984, 222-234. 
MAHRT L., H.L. PAN, A two-layer model of soil hydrology, Boundary Layer Meteorol.,29, 1984, 1-20.

MENDES M. D. et al. Bloqueios Atmosféricos de 1960 a 2000 sobre o Oceano Pacifico Sul: Impactos Climáticos e Mecanismos Físicos Associados, Revista Brasileira de Meteorologia, 20, 2005, 175-190.

MELLOR G.L., YAMADA T., A hierarchy of turbulence closure model for planetary boundary layers. J. Atmos. Sci., 36, 1974, 1722-1735.

MESINGER F., A blocking technique for representation of mountains in atmospheric models. Riv. Meteorol. Aeronaut., 44, 1984, 195-202.

MESINGER, F., et al., The Step-Mountain Coordinate: Model Description and Performance for Cases of Alpine Lee Cyclogenesis and for a Case of an Appalachian Redevelopment. Mon. Wea. Rev., 116, 1988, 14971518 .

NORTE, F., Características del Viento Zonda en la Región de Cuyo. 1988, 220p. Dissertacao de Doutorado. Departamento de Meteorología, Facultad de Ciencias. Exactas y Naturales -Universidad de Buenos Aires. Argentina.

NORTE, F. et al., The Heat Waves in the Center of Argentina. Preprints Sixth International Conference on Southern Hemisphere Meteorology and Oceanography. American Meteorological Society, Chile, April 2000. 198-199.

PADILHA K. C. Estagnação de massa de ar quente e seco sobre a região central do Brasil. 2006, Dissertação de Mestrado. Instituto Nacional de Pesquisas Espaciais.
PAN H.L.,L. MAHRT, Interaction between soil hydrology and boundary-layer development, Boundary Layer Meteorol., 38, 1987, 185-202.

RUSTICUCCI, M., W. VARGAS, Synoptic situations related to spells of extreme temperatures over Argentina. Meteorological Applications 2, 1995, 291-300.

RUSTICUCCI M., BARRUCAND M., Climatologia de Temperaturas extremas en la Argentina. Relación entre la Temperatura Media Estacional y la Ocurrencia de Días Extremos. Meteorológica, 26, 2001, 69-84.

SATYAMURTY P. Rudimentos de Meteorologia Dinâmica, ISBN: 85-17-00019-6, 2004, 154 p.

SELUCHI, M. E., S.Ch Chou, Evaluation of two Eta Model versions for weather forecast over South America, Geofís. Int., 40, 2001, 219-237

SELUCHI M.E., et al. The Northwestern Argentinean Low: A Study of Two Typical Events. Monthly Weather Review - Vol. 131, 2003, 2361-2378.

SELUCHI, M.E., et al. Analysis of three situations of Foehn effect over the Andes (Zonda wind) using the Eta/CPTEC regional model. Wea \& Forec, 18, 2003b, 481-501.

SELUCHI, M. E. et al., Influence of the subtropical Andes on baroclinic disturbances: A cold-front case study. Mon. Wea. Rev, 2006, Em prensa.

VERA C.S. et al., The South American Low-Level Jet Experimint. Bulletin of the American Meteorological Society, 2006, 63-77.

ZHAO, Q., F. H. CARR, A prognostic cloud scheme for operational NWP Models. Mon.Wea. Rev., 125, 1997, 1931-1953. 